\title{
Long Term Neutron Activation in JET DD Operation
}

\author{
Andrej Žohar ${ }^{1,2}$, Igor Lengar ${ }^{1}$, Paola Batistoni ${ }^{3}$, Sean Conroy $^{4}$, Aljaž Čufar ${ }^{1}$, Renata Kierepko ${ }^{5}$, Bor \\ Kos $^{1}$, Stefano Loreti ${ }^{3}$, Jerzy W. Mietelski ${ }^{5}$, Chantal R. Nobs ${ }^{6}$, Lee W. Packer ${ }^{6}$, Mario Pillon ${ }^{3}$, Vladimir \\ Radulović ${ }^{1}$, Marilia I. Savva ${ }^{7}$, Luka Snoj ${ }^{1,2}$, Ion E. Stamatelatos ${ }^{7}$, Žiga Štancar $^{1}$, Theodora \\ Vasilopoulou ${ }^{7}$, Anna Wójcik-Gargula ${ }^{5}$ and JET contributors* \\ ${ }^{1}$ Reactor Physics Department, Jožef Stefan Institute, Jamova cesta 39, 1000 Ljubljana, Slovenia \\ ${ }^{2}$ University of Ljubljana, Faculty of Mathematics and Physics, Jamova cesta 19, 1000 Ljubljana, \\ Slovenia \\ ${ }^{3}$ Department of Fusion and Technologies of Nuclear Safety and Security, ENEA C. R. Frascati, via E. \\ Fermi 45, 00044 Frascati (Roma), Italy \\ ${ }^{4}$ Department of Physics and Astronomy, Uppsala University, Box 516, SE-75120 Uppsala, Sweden \\ ${ }^{5}$ The Henryk Niewodniczanski Institute of Nuclear Physics (IFJ PAN), Polish Academy of Sciences, \\ 31-342 Kraków, Poland \\ ${ }^{6}$ United Kingdom Atomic Energy Authority, Culham Centre for Fusion Energy, Culham Science \\ Centre, Abingdon, Oxon OX14 3DB, UK \\ ${ }^{7}$ INRASTES, NCSR 'Demokritos' 153 10, Agia Paraskevi Attikis, Greece \\ *See the author list of 'Overview of JET results for optimising ITER operation' by J. Mailloux et al. to \\ be published in Nuclear Fusion Special issue: Overview and Summary Papers from the 28th Fusion \\ Energy Conference (Nice, France, 10-15 May 2021)" \\ andrej.zohar@ijs.si
}

\begin{abstract}
In the 2019 C38 Deuterium-Deuterium campaign at JET several different ITER-relevant materials and dosimetry foils were irradiated in a specially designed long-term irradiation station located inside the vacuum vessel with the purpose of testing the activation of ITER materials by fusion neutrons. The samples were exposed to a neutron fluence of $1.9 \mathrm{E} 14 \mathrm{n} / \mathrm{cm}^{2}$ during JET discharges performed in the experimental campaign over a period of 5 months. Gamma ray spectroscopy measurements were performed on irradiated samples to determine the activation of different long-lived isotopes in the samples. Monte Carlo computational analysis was performed to support the experiment by using the measured neutron yield and irradiation time. In this paper we focus on the computational analysis of the dosimetry foils that are used in order to measure the local neutron energy spectrum and flux. The foils were chosen to cover different neutron energies: thus Yttrium and some of the Nickel and Cobalt reactions were used to determine the Deuterium-Tritium fusion fraction, while Scandium and Iron and some of the Nickel and Cobalt reactions were used for comparison of the computed activity with the experimental measurements. The obtained $\mathrm{C} / \mathrm{E}$ values show a reasonably good agreement between calculated and measured activity, thus validating the computational methodology and providing the basis for the analysis of the ITER-relevant materials and future experiments performed at JET in the Deuterium-Tritium campaign.
\end{abstract}

Keywords - JET, Long Term Neutron Irradiation, MCNP

\section{INTRODUCTION}

The neutron irradiation impact on materials is an important research field for the development of fusion technologies. The Joint European Torus (JET) tokamak is currently the only tokamak capable of performing fusion plasma experiments with Deuterium-Deuterium (DD), Tritium-Tritium (TT) and also Deuterium-Tritium (DT) fusion reactions, thus producing high 2.45 MeV and 14.1 MeV neutron fluence. The high neutron fluence can be used to perform neutron irradiation experiments of different materials, of which the most important are materials that will be used in components of the ITER tokamak.

To support ITER and study the impact of neutron irradiation on materials, especially for DT plasmas, several experiments are planned to be performed in the upcoming TT and DT experiential campaigns at JET planned to commence in $2021 / 22$. In preparation for the DT campaign at JET, several important experiments have already been performed [1], such as $14 \mathrm{MeV}$ neutron calibration [2,3], neutronics benchmark experiments [4-8] and activation measurement experiments [911]. For the preparation of experiments on the neutron induced activation on materials, samples of the main components of the ITER tokamak and dosimetry foils were irradiated during the 2019 C38 Deuterium experimental campaign at JET [12,13]. Samples were irradiated in a long-term irradiation station (LTIS) located close to the plasma boundary for several 
months. The goal of the experiment was to study the DD neutron activation of ITER materials and preparation of the experimental methodology for the upcoming TT and DT experimental campaigns. The developed methodology consists of the execution of irradiation experiments, measurements of sample activation and computational support to the experiment.

To properly evaluate the activation of different isotopes in the ITER samples and validate the computational methodology, several different dosimetry foils (Cobalt, Nickel, Iron, Yttrium, and Scandium) were irradiated together with the ITER samples in the LTIS. The dosimetry foils were chosen to cover different neutron energy regions, from thermal neutrons (energies up to $1 \mathrm{eV}$ ) up to fast neutrons (from $0.1 \mathrm{MeV}$ to $14 \mathrm{MeV}$ ). In this paper, the comparison between experimental measurements of irradiated dosimetry foil activities and calculated activities will be presented.

The paper is organized as follows. In the first section, the irradiation experiment is presented. Key aspects concerning the experiment are: the long-term irradiation station together with different ITER samples and dosimetry foils, the conditions to which samples in the LTIS were exposed during tokamak operation and the measurements of sample activity performed after irradiation. In the second section, the computational methodology is presented. The methodology consists of a detailed and validated JET Monte Carlo N-Particle transport code (MCNP [14]) model and the procedure to calculate the isotope activities in dosimetry foils at the end of the irradiation. In the last section the results of activity measurements for dosimetry foils will be compared with calculated activities for validation of the computation methodology for upcoming TT and DT irradiation experiments at JET.

\section{EXPERIMENTAL MEASUREMENTS}

\section{A. Long-term irradiation station}

For the neutron irradiation impact on materials experiment in the DD, TT and DT campaigns at JET, a new LTIS was made. The LTIS was designed to hold several different samples of different sizes and be positioned in one of the irradiation positions located close to the plasma boundary but not within the vacuum vessel. The chosen position allows the samples to be exposed to high neutron fluence and be removed between experimental campaigns at JET without breaching the vacuum barrier. The LTIS position inside the JET vacuum vessel is presented in Fig. 1. For LTIS to hold samples of different size, two holders were made caller 'RADA' holder and 'ACT' holder. The 'ACT' holder has 26 equal size positions in which many samples can be inserted simultaneously and photo of the 'ACT' holder is presented in Fig. 2. The 'RADA' holder has 28 positions of different sizes.

For the experiment performed in the C38 deuterium campaign, the 'ACT' holder in the LTIS was loaded with 27 ITER material samples and 70 samples of different dosimetry foils. The thicknesses of the samples varied from $0.1 \mathrm{~mm}$ to 1 $\mathrm{mm}$ with the majority of samples being $0.5 \mathrm{~mm}$ thick. Fig. 2 shows the 'ACT' holder loaded with different samples for the irradiation experiment. The irradiated ITER material samples include material samples of poloidal field coil jacket, toroidal field coil radial closure plate steels, EUROFER 97-2 steel, Tungsten and $\mathrm{CuCrZr}$ materials from the divertor, Inconel 718, $\mathrm{CuCrZr}$ and $316 \mathrm{~L}$ stainless steel for the blanket modules and vacuum vessel forging samples [13].

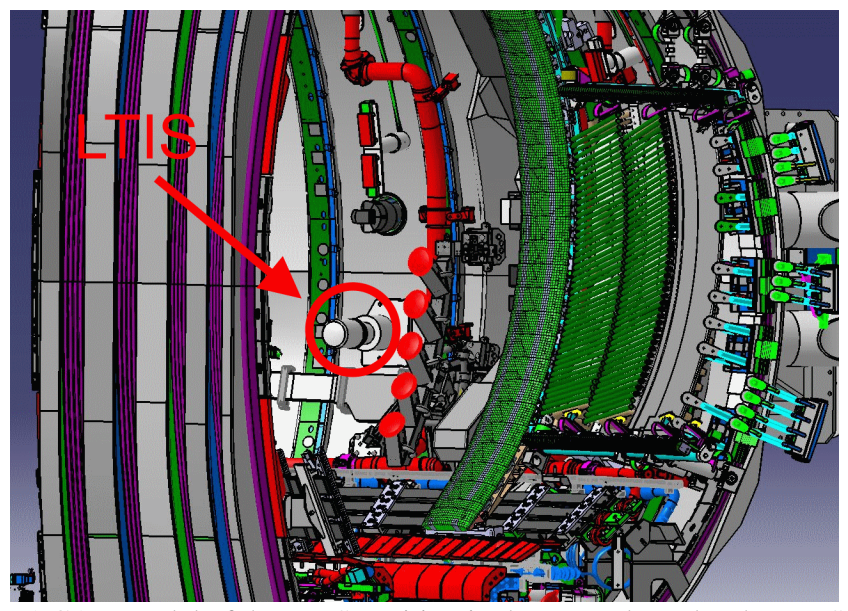

Fig. 1 CAD model of the LTIS position in the JET tokamak. The LTIS is located in the $7^{\text {th }}$ octant below the middle plane of the tokamak.

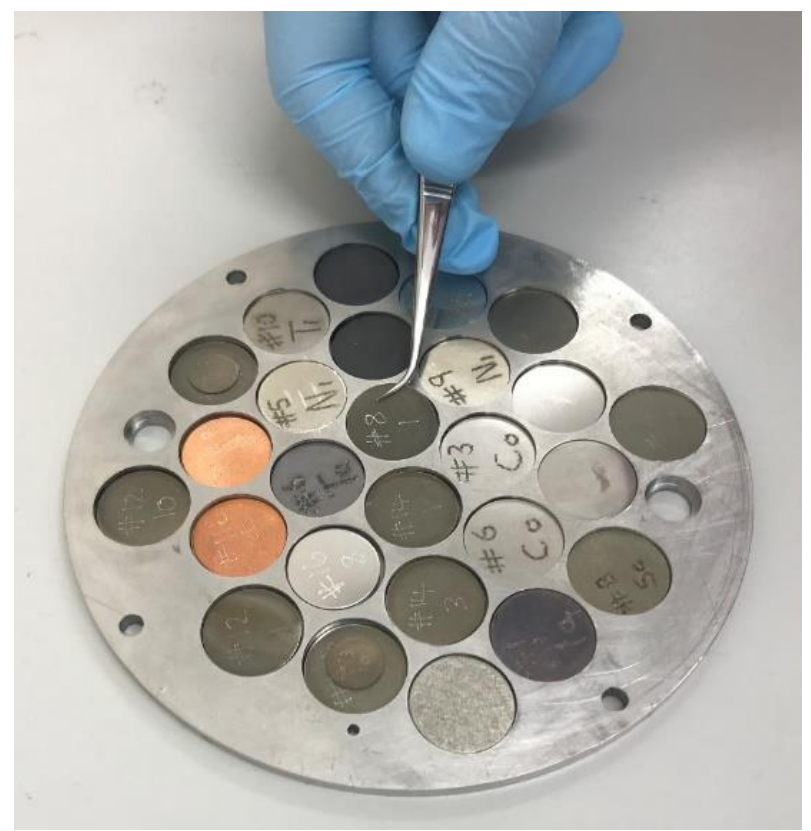

Fig. 2 Photograph of the outer long-term irradiation station sample holder loaded with ITER samples and dosimetry foils. The samples were irradiated in the $\mathrm{C} 38$ deuterium campaign at JET.

The dosimetry foils in LTIS were chosen to cover different neutron energy regions, from thermal neutrons to fast neutrons. The dosimetry foils used in the irradiation experiment were made from Cobalt, Nickel, Iron, Scandium and Yttrium.

\section{B. Experimental condition and gamma ray spectroscopy measurements}

The LTIS was installed on $27^{\text {th }}$ July 2019 . The samples were irradiated in the C38 campaign from $29^{\text {th }}$ July 2019 till $20^{\text {th }}$ December 2019. During the campaign, there were 101 days of irradiation and 46 days of no experimental irradiation. The neutron irradiation during plasma discharges was short $(10 \mathrm{~s}$ to 
20 s per plasma discharge) with cooling time in between discharges. The total neutron yield of the C38 experimental campaign at JET was $3.15 \cdot 10^{19}$ neutrons. The neutron fluence the samples in LTIS were exposed to during the C38 experimental campaign was $1.9 \cdot 10^{14} \mathrm{n} / \mathrm{cm}^{2}$.

The samples from LTIS were retrieved on $3^{\text {rd }}$ January 2020 , approximately 13 days after the end of irradiation. The ITER samples and dosimetry foils were sent to different European laboratories for analysis: CCFE, ENEA, IFJ-PAN and NCSRD. All laboratories performed high resolution gamma ray measurements of samples with similar methodologies for calibration, analysis and reporting of the sample activity results. The reported measured activity of all isotopes in samples were decay-corrected to the end of the JET irradiation period $-20^{\text {th }}$ December 2019 at 21:30:00 GMT. Besides decay-corrected activities, all laboratories also reported sample mass and measurement uncertainty. A detailed description of the measurement process and obtained results for ITER samples are presented in a separate paper [13].

\section{SAMPLE ACTIVATION CALCULATION METHODOLOGY}

The computational support for the neutron activation of materials in the LTIS was performed with the MCNP (version 6.2 [14]) code together with the nuclear data library FENDL3.1d [15]. The computations were performed with an already existent MCNP model of the tokamak JET, which has been validated on several experiments in the past at JET $[2,3,4,8]$. The model contains all larger components inside the vacuum vessel with slight simplifications with the aim to omit the use of toroidal surfaces in the description of components. The reason is that in MCNP models, containing toroidal surfaces, the computations take on average longer than in models without torus surfaces for the same number of simulated particles. The outside of the tokamak structure is partly defined with a low

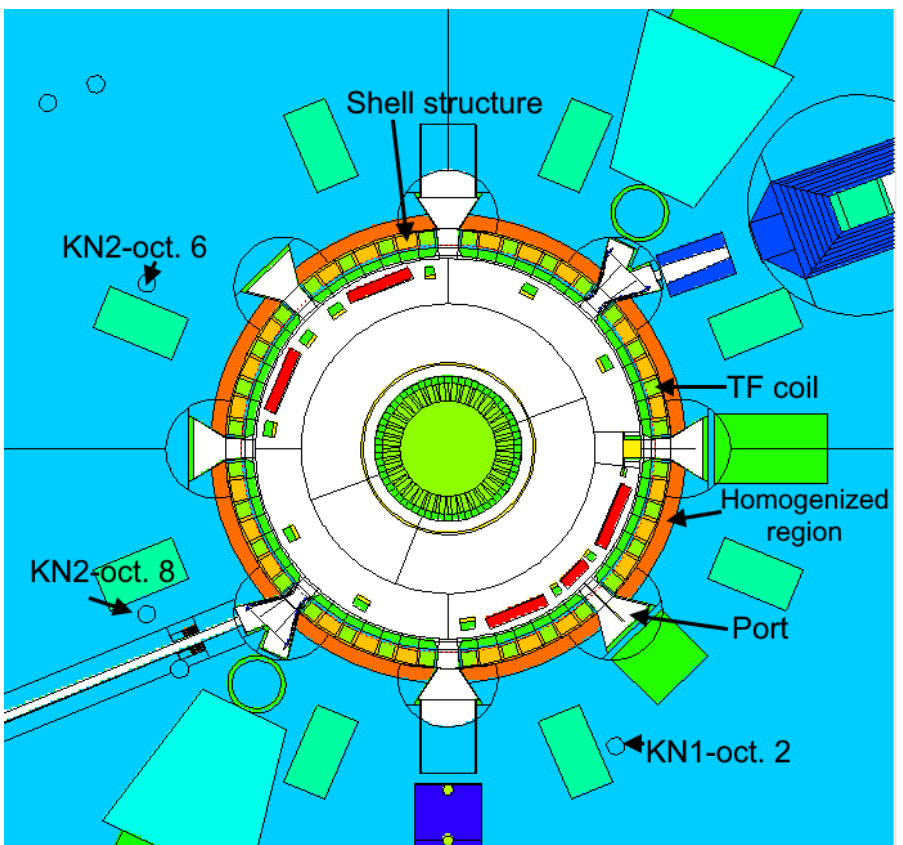

density mixture of Inconel with additions, representing the equipment surrounding the torus (homogenized surrounding region) and some of the ex-vessel diagnostic equipment. The drawing of the MCNP model is presented in Fig. 3.

The neutron emissivity profile used for calculations was taken from a pre-computed TRANSP plasma transport simulation for a NBI heated JET plasma [16,17]. As in the DD plasma some DT neutrons are produced due to the burnup of tritium generated in the DD reactions, therefore the neutron source used for computational support was a combination of DD and DT neutron sources [18]. The neutron spectrum for DD and DT plasma used in the analysis was assumed to be Maxwellian, computed for a plasma with average ion temperatures of $\mathrm{T}_{\mathrm{th}}=20 \mathrm{keV}$. All of the neutrons were presumed to be born isotropically in direction.

\section{A. LTIS MCNP model}

To properly support the neutron activation experiment a detailed model of the LTIS was added to the validated MCNP model of the JET tokamak. The MCNP model was constructed from the CATIA model with the help of GRASP (GrasshopperRhino analyzing surfaces for MCNP) which was developed at the Reactor physics department of the Jožef Stefan Institute [19]. In the model of the LTIS all details were preserved and all irradiation position were filled with specified samples. Fig. 4 represents the MCNP model of LTIS used in the analysis. The bulky part of the LTIS is facing the plasma and the RADA holder is positioned closer to the plasma compared to the ACT holder.

\section{B. Activity computation methodology}

For comparison with measurements the activity of different isotopes in the dosimetry foils can be calculated using the equation [20,21]:

$$
A=R\left(1-e^{-\lambda t_{i r r}}\right) e^{-\lambda t_{c o o l}}
$$

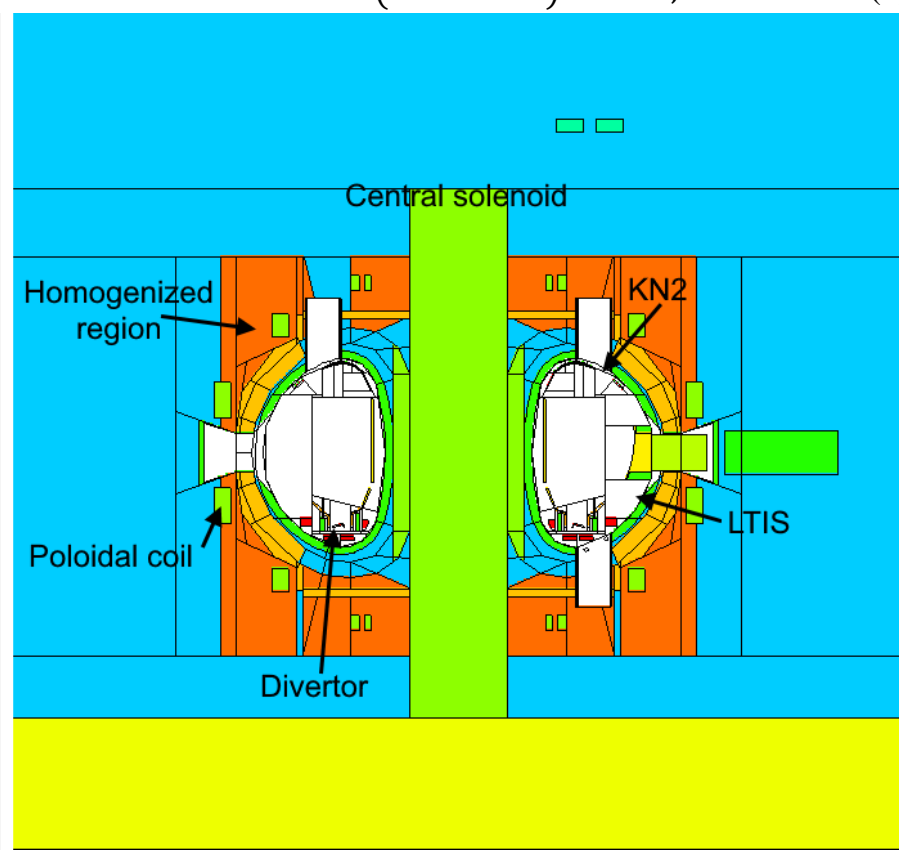

Fig. 3 Section view of the MCNP model of the tokamak JET used for computation support for the LTIS experiment. In the right figure the approximate location of the LTIS is marked. 


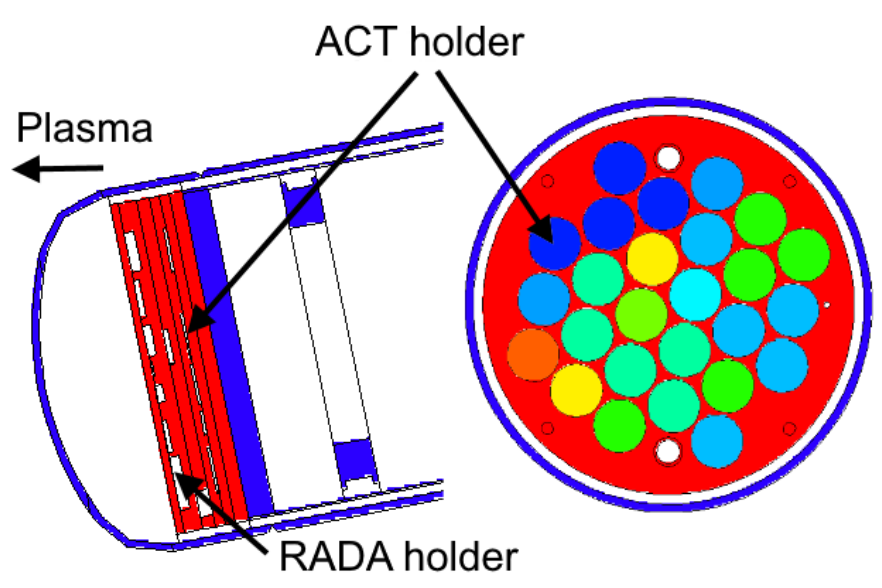

Fig. 4 MCNP model of the LTIS with marked ACT and RADA holder and direction of plasma. The ACT holder is filled with different ITER samples and dosimetry foils.

where $R$ is the calculated reaction rate for the studied isotope in the dosimetry foil, $\lambda$ is the decay constant of the activated isotope, $t_{i r r}$ is the irradiation time of the samples and $t_{\text {cool }}$ is the cooling time. As isotopes Co-58 (half-life 70.86 days) and Sc-46 (half-life 83.79 days) have a relatively short half-life the experimental campaign cannot be represented in calculations as a continuous irradiation of 101 days followed by 46 days of cooling. To properly calculate the activity of isotopes at the end of C38 deuterium campaign the irradiation and cooling days were merged on weekly basis - 22 weeks of irradiation. The final activity of samples is calculated using the equation (1) plus the decay of activated isotopes produced in previous weeks. The equation used can be thus written as:

$$
A_{n}=R\left(1-e^{-\lambda t_{i r r}}\right) e^{-\lambda t_{c o o l}}+A_{n-1} e^{-\lambda t_{w}},
$$

where $n$ is the number of the week and $t_{w}$ is time in one week. For a more accurate calculation the last day was simulated without any merger of pulses.

The reaction rates were calculated using MCNP in the modelled samples in the LTIS. The analysis presented in this paper focused on reactions presented in Table I. The cross sections for calculation of reaction rates were taken from the IRDFF-II [22] nuclear data library and JEFF-3.3 [23] nuclear data library for reactions missing in the IRDFF-II nuclear data library (reaction $\mathrm{Ni}-58(\mathrm{n}, \mathrm{np}) \mathrm{Co}-57$ ).

$$
\text { Table I }
$$

Studied reactions in the dosimetry foils from LTIS in the C38 deuterium campaign at JET.

\begin{tabular}{|l|c|}
\hline $\begin{array}{l}\text { Dosimetry foil } \\
\text { material }\end{array}$ & Reaction \\
\hline Cobalt & Co-59(n, $\gamma)$ Co-60 \\
\cline { 2 - 2 } & Co-59(n,2n)Co-58 \\
\hline \multirow{2}{*}{ Nickel } & Ni-58(n,p)Co-58 \\
\cline { 2 - 2 } & Ni-60(n,p)Co-60 \\
\cline { 2 - 2 } & Ni-58(n,np)Co-57 \\
\hline Iron & Fe-54(n,p)Mn-54 \\
\hline Scandium & $\mathrm{Sc}-45(\mathrm{n}, \gamma)$ Sc-46 \\
\hline Yttrium & $\mathrm{Y}-89(\mathrm{n}, 2 \mathrm{n}) \mathrm{Y}-88$ \\
\hline
\end{tabular}

Despite the LTIS position being close to the neutron source and due to the very small volume of tally regions, variance reduction method was used to reduce the Monte Carlo statistical uncertainty in the thermal region of the neutron spectra for activation reactions produced predominantly by thermal neutrons (Co-59(n, $\gamma)$ Co-60). This was achieved using weight windows generated by the code ADVANTG [24].

\section{COMPARISON OF CALCULATED AND MEASURED ACTIVITY}

\section{A. Fast DT neutron reactions}

As mentioned, the dosimetry reactions were chosen to cover different regions of the neutron spectra. The reactions Co59(n,2n)Co-58, Ni-60(n,p)Co-60, Ni-58(n,np)Co-57 and Y89(n,2n)Y-88 have a threshold energy above the energy of DD neutrons $(2.45 \mathrm{MeV})$. Due to this, they can only be a result of interaction with fast DT neutrons $(14.1 \mathrm{MeV})$ that are produced in the DD plasma due to tritium production in DD reactions. Thus, the analysis of these reactions represent an opportunity to determine the DT neutron fraction in the DD plasma. The cross sections for the studied reactions from the IRDFF-II and JEFF3.3 nuclear data libraries are presented in Fig. 5.

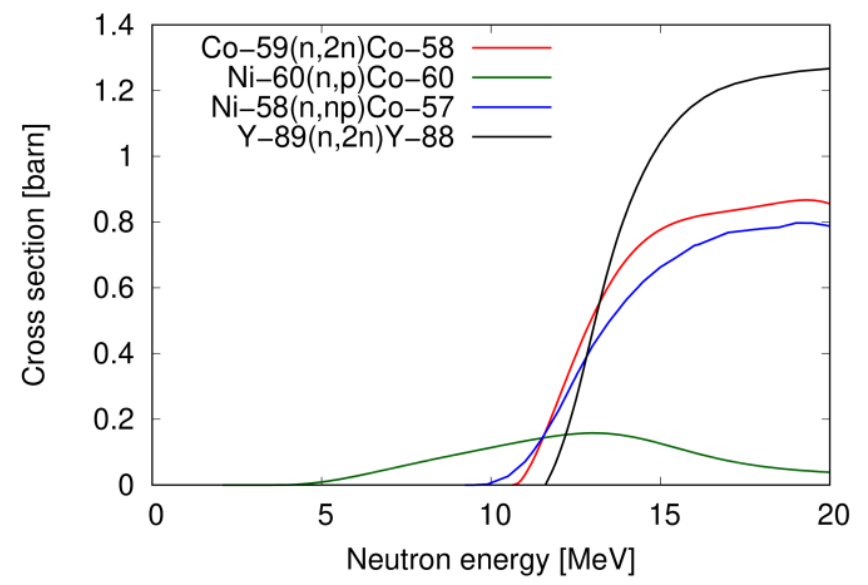

Fig. 5 Cross sections for neutron reactions with high threshold energies. The reaction Ni-58(n,np)Co-57 cross section is taken from the JEFF-3.3 nuclear data library while other cross sections are taken from the IRDFF-II nuclear data library.

In general a $1 \%$ DT neutron fraction in a DD plasma is assumed. However, the $\mathrm{C} / \mathrm{E}$ values for the activities of isotopes produced by fast reactions were below 1 in all cases. Due to this, the DT fraction was varied to obtain a better agreement between measurements and computations. The analysis showed that a $1.4 \%$ DT neutron fraction produced the calculated-tomeasured activity ratios closest to 1 . The $\mathrm{C} / \mathrm{E}$ values for $1.4 \%$ DT neutron fraction for isotopes produced by fast DT neutrons are presented in Fig. 6.

The uncertainties in the $\mathrm{C} / \mathrm{E}$ results presented in Fig. 6 were computed from the 2 sigma statistical uncertainties of MCNP calculations, uncertainties in measured sample activity and uncertainty of the neutron yield. The statistical uncertainty for all calculated reaction rates with MCNP was below $2 \%$. The major part of the uncertainty of the $\mathrm{C} / \mathrm{E}$ values are thus due to the measurement uncertainties and neutron yield uncertainty.

The C/E values presented in Fig. 6 vary from 0.9 to 1.6 for reactions sensitive to fast DT neutrons, assuming the total neutron yield includes $1.4 \%$ DT neutrons. The most significant difference between calculations and measurements is observed for cobalt samples measured by IFJ. The dispersion in the $\mathrm{C} / \mathrm{E}$ 


\section{Reactions produced by only fast DT neutrons}

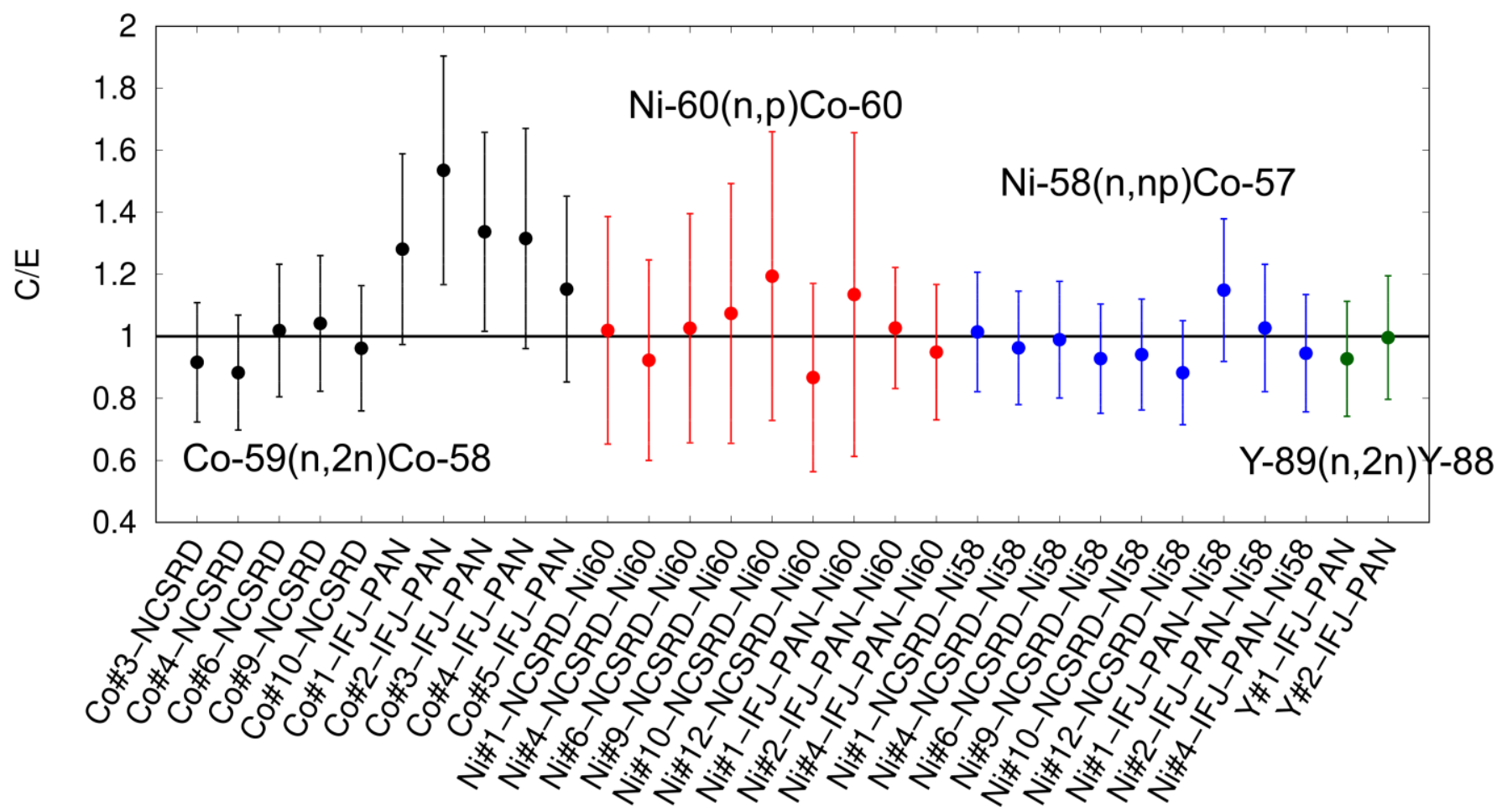

Fig. 6 Comparison of the calculated activity with measured for isotopes in dosimetry foils produced by fast neutrons - Co-59(n,2n)Co-58, Ni$60(n, p) C o-60$, Ni-58(n,np)Co-57 and Y-89(n,2n)Y-88. The DT neutron fraction used in the analysis was $1.4 \%$. The error bars represent 2 sigma statistical uncertainty of MCNP calculations and uncertainty in measured sample activity. The number next to the material name represents the ID of the sample.

values presented in Fig. 6 can be attributed to the differences in the measurement methodology at different institutions and different size of samples measured by institutions. Despite the variations in the $\mathrm{C} / \mathrm{E}$ values the computed activities are in reasonably good agreement with the measured activity by different institutions. The determined $1.4 \%$ DT fraction was used in further analyses of reactions produced by DD and DT neutrons.

\section{B. DD and DT neutron reactions}

The reactions studied on isotopes, activated by both DD and DT neutrons were Co-59(n, $\gamma) \mathrm{Co}-60$, Ni-58(n,p)Co-58, Fe54(n,p)Mn-54 and Sc-45(n, $\gamma)$ Sc-46. The cross sections for the reactions from the IRDFF-II are depicted in Fig. 7. Reactions $\mathrm{Ni}-58(\mathrm{n}, \mathrm{p}) \mathrm{Co}-58$ and Fe-54(n,p)Mn-54 are threshold reactions with thresholds at $0.8 \mathrm{MeV}$ and $0.7 \mathrm{MeV}$ while the Co$59(\mathrm{n}, \gamma) \mathrm{Co}-60$ and $\mathrm{Sc}-45(\mathrm{n}, \gamma) \mathrm{Sc}-46$ reactions are produced predominantly by thermal neutrons.

The $\mathrm{C} / \mathrm{E}$ values for these reactions are presented in Fig. 8. As in the case of solely DT neutron reactions the uncertainties in the $\mathrm{C} / \mathrm{E}$ values were computed from the 2 sigma statistical uncertainties of MCNP calculations, uncertainties in measured sample activity and uncertainty of the neutron yield. The MCNP statistical uncertainty for all calculated reaction rates with MCNP was below $2 \%$. The major part of the uncertainty of the $\mathrm{C} / \mathrm{E}$ values are thus due to the measurement uncertainty and neutron yield uncertainty.

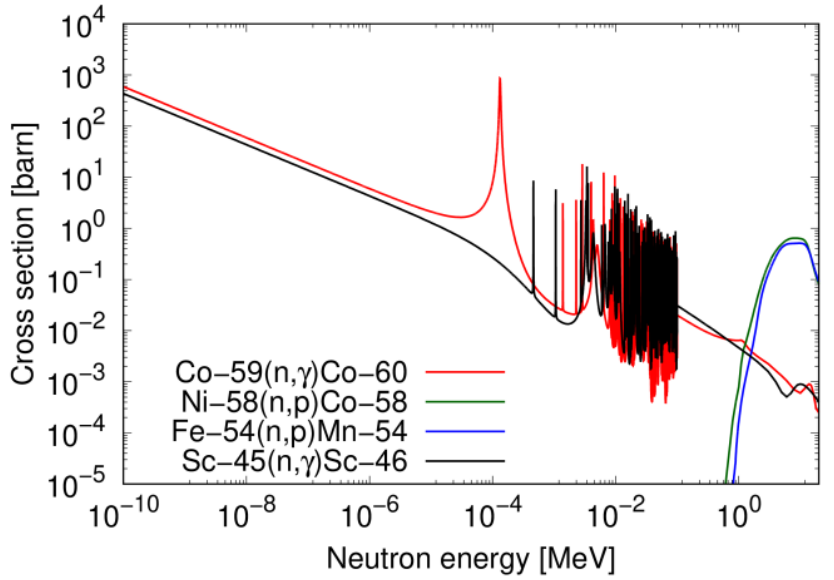

Fig. 7 Cross sections for reactions produced by DD and DT neutrons. All cross sections are taken from the IRDFF-II nuclear data library.

For the reactions, predominantly produced by thermal neutrons, the $\mathrm{C} / \mathrm{E}$ values vary for cobalt samples measured by two different institutions. For the samples measured by the NCSRD show C/E values around 1, while the cobalt samples measured by IFJ show higher $\mathrm{C} / \mathrm{E}$ values of around 1.5 similar to results presented in Fig. 6. Sc samples exhibit $\mathrm{C} / \mathrm{E}$ values of around 1.3. As in the case of DT neutron activation reactions the Co samples measured NCSRD and IFJ were of different size. 


\section{Reactions produced by DD and DT neutrons}

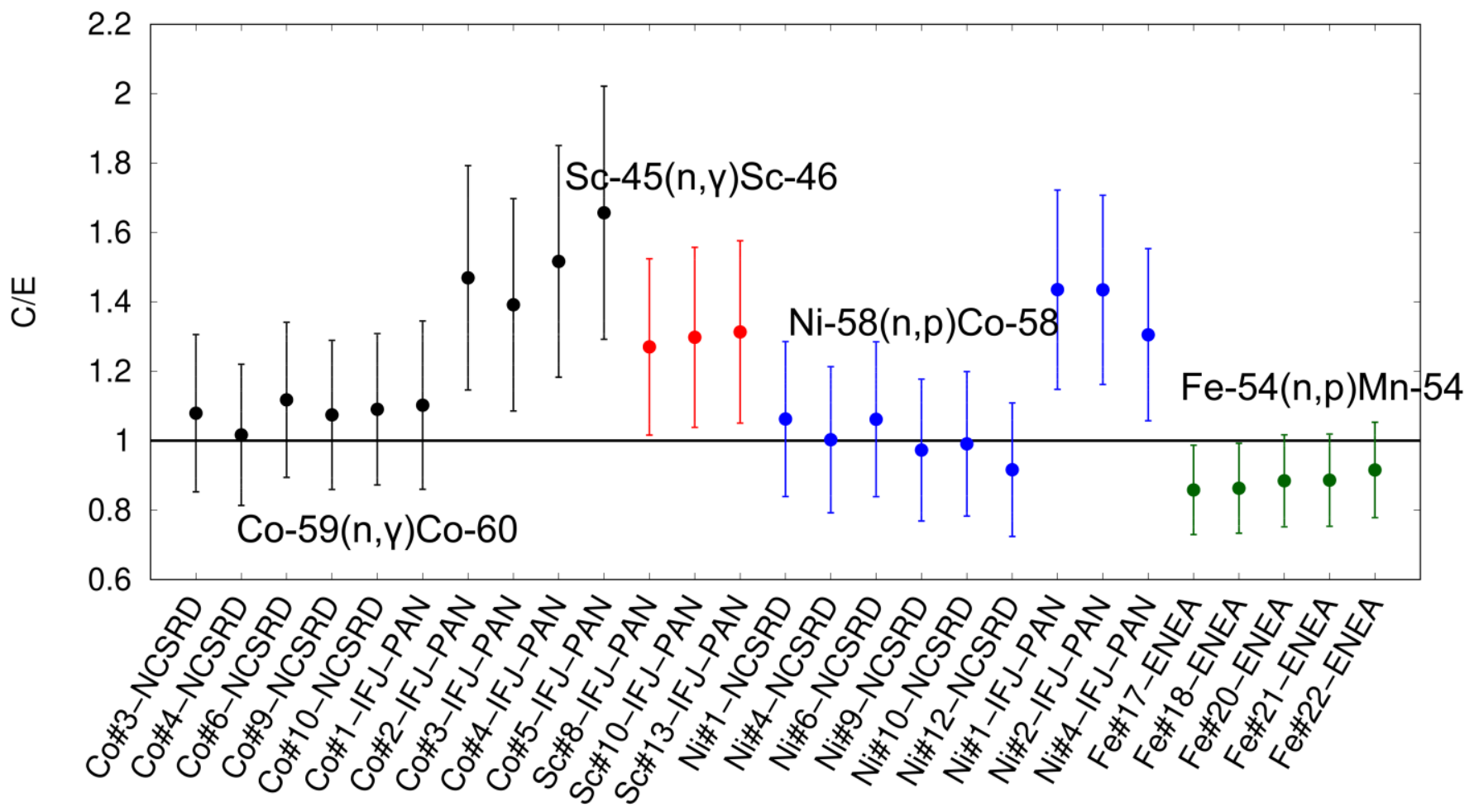

Fig. 8 Comparison of the calculated activity with measured isotopes in dosimetry foils produced by DD and DT neutrons - Co-59(n, $\gamma$ )Co- 60 , Sc45(n, $)$ Sc-46, Ni-58(n,p)Co-58 and Fe-54(n,p)Mn-54. The DT neutron fraction used in the analysis was $1.4 \%$. The error bars represent 2 sigma statistical uncertainty of MCNP calculations and uncertainty in measured sample activity. The number next to the material name represents the ID of the sample.

For the Ni-58(n,p)Co-58 reaction measured by NCSRD the $\mathrm{C} / \mathrm{E}$ values are around 1 . The $\mathrm{C} / \mathrm{E}$ values for nickel samples measured by IFJ are around 1.4. The $\mathrm{C} / \mathrm{E}$ values for iron samples measured by ENEA are around 0.9. An analysis is currently under way with the aim to explain the differences in the $\mathrm{C} / \mathrm{E}$ values between different institutions.

Despite the variations in the $\mathrm{C} / \mathrm{E}$ values the computed activities are in reasonably good agreement with the measured activity by different institutions.

\section{CONCLUSIONS}

The JET tokamak currently offers one of the most suitable environments to study the effects of fusion neutron irradiation in materials. In the 2019 C38 deuterium experimental campaign several different ITER material samples and dosimetry foils were irradiated in the long-term irradiation station. The samples were exposed to a neutron fluence of $1.9 \cdot 10^{14} \mathrm{n} / \mathrm{cm}^{2}$ during JET discharges performed in the experimental campaign over a period of 5 months.

Gamma ray spectroscopy measurements were performed on irradiated samples by several different European laboratories to determine the activation of different long-lived isotopes in the samples.

The computational methodology for calculation of activation of isotopes in different samples was performed with the MCNP code. To properly simulate activation, a detailed model of the LTIS was made. All dosimetry foils in the ACT holder were modelled and for each sample reaction rates in the sample were calculated. The activities for the different isotopes at the time the holder was removed from JET were calculated taking into account the measured neutron yield and irradiation time.

The calculated values were compared to the measured. The $\mathrm{C} / \mathrm{E}$ values vary from 0.9 to 1.6 for reactions sensitive to only fast DT neutrons, assuming the total neutron yield includes 1.4 $\%$ DT neutrons. For reactions produced by DD and DT neutrons the $\mathrm{C} / \mathrm{E}$ values vary from 0.9 to 1.6 .

Despite the variations in the $\mathrm{C} / \mathrm{E}$ values, the computed activities of isotopes in samples are in a reasonably good agreement with the measured activities by different institutions within the total uncertainties, thus validating the computational methodology and providing the basis for analysis of the ITER relevant materials and future experiments performed at JET in the Tritium-Tritium and Deuterium-Tritium campaign. Additional analysis is being performed with the aim to explain the differences in the $\mathrm{C} / \mathrm{E}$ values between different institutions.

\section{ACKNOWLEDGMENT}

This work has been carried out within the framework of the EUROfusion Consortium and has received funding from the Euratom research and training programme 2014-2018 and 2019-2020 under grant agreement No 633053. The views and 
opinions expressed herein do not necessarily reflect those of the European Commission.

The work was supported by the Slovenian Ministry of Education, Science and Sport (projects codes: P2-0073 Reactor Physics; P2-0405 Fusion technologies; 1000-17-0106-6 Training of young researchers).

\section{REFERENCES}

[1] E. Joffrin, et al., "Overview of the JET preparation for deuterium-tritium operation with the ITER like-wall”, Nuclear Fusion, vol. 59, 112021, 2019.

[2] A. Ćufar, et al., "Calculations to support In situ neutron yield calibrations at the joint European torus", Fusion science and technology, vol. 74, 370314, 2018.

[3] P. Batistoni, "On the absolute calibration of neutron measurements in fusion reactors", Fusion Engineering and Design, vol, 105, 58-69, 2016.

[4] P. Batistoni, et al., "Benchmark experiments on neutron streaming through JET Torus Hall penetrations", Nuclear Fusion, vol. 55, 042017, 2015.

[5] R. Villari, et al., "ITER oriented neutronics benchmark experiments on neutron streaming and shutdown dose rate at JET", Fusion Engineering and Design, vol 123, 171-176, 2017.

[6] T. Vasilopoulou, et al., "Neutron streaming along ducts and labyrinths at the JET biological shielding: Effect of concrete composition", Radiation Physics and Chemistry, vol. 116, 359-364, 2015.

[7] J. Naish, et al., "Comparison of neutron flux streaming calculations to the 2019-2020 JET experimental deuterium-deuterium results", Fusion Engineering and Design, vol. 170, 112538, 2021.

[8] B. Kos, et al., "Application of ADVANTG to the JET3 - NEXP streaming benchmark experiment", Fusion Engineering and Design, vol.147, 1-10, 2019.

[9] I. Lengar, et al., "Characterization of JET neutron field in irradiation locations for DD, DT and TT plasmas", Fusion Engineering and Design, vol. 146, 1967-1970, 2019.

[10] L.W. Packer, et al., "Status of ITER material activation experiments at JET”, Fusion Engineering and Design, vol. 124, 1150-1155, 2017.

[11] G. Stankunas, et al., "Analysis of activation and damage of ITER material samples expected from DD/DT campaign at JET", Fusion Engineering and Design, vol. 125, 307-313, 2017.

[12] L.W. Packer, et al., "Activation of ITER materials in JET: nuclear characterization experiments for the long-term irradiation station", Nuclear fusion, vol. 58, 096013, 2018.

[13] L.W. Packer, et al., "Technological exploitation of the JET neutron environment: progress in ITER materials irradiation and nuclear analysis", to be published in Nuclear Fusion.

[14] C.J. Werner, "MCNP User Manual - Code Version 6.2", Los Alamos National Laboratory, report La-UR-17-29981, 2017.

[15] IAEA Fusion Evaluated Nuclear Data Library - FENDL-3.1d, International Atomic Energy Agency, https://www-nds.iaea.org/fendl/

[16] J. Breslau, et al. "TRANSP”, 2018

[17] Ž. Štancar, et al. "Multiphysics approach to plasma neutron source modelling at the JET tokamak", Nuclear Fusion, vol 59, 096020, 2019.

[18] S. Conroy, et al., "Time resolved measurements of triton burnup in JET plasmas", Nuclear Fusion, vol. 28, 2127, 1988.

[19] B. Kos and L. Snoj, "On using Grasshopper add-on for CAD to MCNP conversion", in PHYSOR, Sun Valley, USA, 2016.

[20] A. Žohar and L. Snoj, "On the dose fields due to activated cooling water in nuclear facilities", Progress in Nuclear energy, vol. 177, 103042, 2019.

[21] A. Žohar, et al., "Water activation experiments and calculations at JSI TRIGA research reactor", Proceedings: NENE2020: 29th International Conference Nuclear Energy for New Europe, 2020.

[22] A. Trkov, et al. "IRDFF-II: A New Neutron Metrology Library", Nuclear Data Sheets, vol. 163, 1-108, 2020.

[23] A.J.M. Plompen, et al., "The joint evaluated fission and fusion nuclear data library, JEFF-3.3", The European Physics Jurnal, vol. 56, 181, 2020.

[24] S.W. Mosher, "ADVANTG-An Autometed Variance Reduction Parameter Generator,” ORNL/TM-2013/416 Rev.1, Oak Ridge National Laboratory, 2015. 